Editorial

\title{
Innovations in nature inspired optimization and learning methods
}

The nine papers included in this special issue represent a selection of extended contributions presented at the Third World Congress on Nature and Biologically Inspired Computing (NaBIC2011), held in Salamanca, Spain, October 19-21, 2011.

Papers were selected on the basis of fundamental ideas and concepts rather than the direct usage of well-established techniques. This special issue is then aimed at practitioners, researchers and postgraduate students, who are engaged in developing and applying, advanced Nature and Biologically Inspired Computing Models to solving real-world problems. The papers are organized as follows.

The first paper by Apeh et al. present a comparative investigation of 4 approaches for classifying dynamic customer profiles built using evolving transactional data over time. The changing class values of the customer profiles were analyzed together with the challenging problem of deciding whether to change the class label or adapt the classifier. The results from the experiments conducted on a highly sparse and skewed real-world transactional data show that adapting the classifiers leads to more stable classification of customer profiles in the shorter time windows; while relabelling the changed customer profile classes leads to more accurate and stable classification in the longer time windows.

Frolov et al. suggested in the second paper a new approach to Boolean factor analysis, which is an extension of the previously proposed Boolean factor analysis method: Hopfield-like attractor neural network with increasing activity. The authors increased its applicability and robustness when complementing this method by a maximization of the learning set likelihood function defined according to the Noisy-OR generative model. They demonstrated the efficiency of the new method using the data set generated according to the model. Successful application of the method to the real data is shown when analyzing the data from the Kyoto Encyclopedia of Genes and Genomes database which contains full genome sequencing for 1368 organisms.

In the sequel, Triguero et al. analyze the integration of a wide variety of noise filters into the self-training process to distinguish the most relevant features of filters. They are focused on the nearest neighbour rule as a base classifier and ten different noise filters. Then, they provide an extensive analysis of the performance of these filters considering different ratios of labelled data. The results are contrasted with nonparametric statistical tests that allow us to identify relevant filters, and their main characteristics, in the field of semi-supervised learning.

In the Fourth paper, Gutiérrez-Avilés et al. present the TriGen algorithm, a genetic algorithm that finds triclusters of gene expression that take into account the experimental conditions and the time points simultaneously. The authors have used TriGen to mine datasets related to synthetic data, yeast (Saccharomyces Cerevisiae) cell cycle and human inflammation and host response to injury experiments. TriGen has proved to be capable of extracting groups of genes with similar patterns in subsets of conditions and times, and these groups have shown to be related in terms of their functional annotations extracted from the Gene Ontology project.

In the following paper, Varela et al. introduce and study the application of Constrained Sampling Evolutionary Algorithms in the framework of an UAV based search and rescue scenario. These algorithms have been developed as a way to harness the power of Evolutionary Algorithms (EA) when operating in complex, noisy, multimodal optimization problems and transfer the advantages of their approach to real time real world problems that can be transformed into search and optimization challenges. These types of problems are denoted as Constrained Sampling problems and are characterized by the fact that the physical limitations of reality do not allow for an instantaneous determination of the fitness of the points present in the population that must be evolved. A general approach to address these problems is presented and a particular implementation using Differential Evolution as an example of CS-EA is created and evaluated using teams of UAVs in search and rescue missions.The results are compared to those of a Swarm Intelligence based strategy in the same type of problem as this approach has been widely used within the UAV pathplanning field in different variants by many authors.

In the Sixth paper, Zhao et al. introduce human intelligence into the computational intelligent algorithms, namely particle swarm optimization (PSO) and immune algorithms (IA). A novel humancomputer cooperative PSO-based immune algorithm (HCPSO-IA) is proposed, in which the initial population consists of the initial artificial individuals supplied by human and the initial algorithm individuals are generated by a chaotic strategy. Some new artificial individuals are introduced to replace the inferior individuals of the population. HCPSO-IA benefits by giving free rein to the talents of designers and computers, and contributes to solving complex layout design problems. The experimental results illustrate that the proposed algorithm is feasible and effective.

In the sequel, Rebollo-Ruiz and Graña give an extensive empirical evaluation of the innovative nature inspired Gravitational Swarm Intelligence (GSI) algorithm solving the Graph Coloring Problem (GCP). GSI follows Swarm Intelligence problem solving approach, where spatial position of agents are interpreted as problem solutions and agent motion is determined solely by local information, avoiding any central control system. To apply GSI to search for solutions of GCP, the authors map agents to graph's nodes. Agents move as particles in the gravitational field defined by goal objects corresponding to colors. When the agents fall in the gravitational well of the color goal, their corresponding nodes are colored by this 
color. Graph's connectivity is mapped into a repulsive force between agents corresponding to adjacent nodes. The authors discuss the convergence of the algorithm by testing it over a extensive suite of well-known benchmarking graphs. Comparison of this approach to state-of-the-art approaches in the literature show improvements in many of the benchmark graphs.

In the Eighth paper, Macăs et al. demonstrates how the novel algorithms can be derived from opinion formation models and empirically demonstrates their usability in the area of binary optimization. Particularly, it introduces a general SITO algorithmic framework and describes four algorithms based on this general framework. Recent applications of these algorithms to pattern recognition in electronic nose, electronic tongue, newborn EEG and ICU patient mortality prediction are discussed. Finally, an open source SITO library for MATLAB and JAVA is introduced.

In the final paper, Madureira et al. present a negotiation mechanism for dynamic scheduling based on social and collective intelligence. Under the proposed negotiation mechanism, agents must interact and collaborate in order to improve the global schedule. Swarm Intelligence is considered a general aggregation term for several computational techniques which use ideas and get inspiration from the social behaviors of insects and other biological systems. This work is concerned with negotiation, where multiple self-interested agents can reach agreement over the exchange of operations on competitive resources. A computational study was performed in order to validate the influence of negotiation mechanism in the system performance and the SI technique. From the obtained results it was possible to conclude about statistical evidence that negotiation mechanism influence significantly the overall system performance and about advantage of Artificial Bee Colony on effectiveness of makespan minimization and on the machine occupation maximization.

We would like to thank our peer-reviewers for their diligent work and efficient efforts.We are also grateful to the Editor-inChief of Neurocomputing, Prof. Tom Heskes, for his continued support for the NABIC conference and for the opportunity to organize this Special issue.

\section{Acknowledgments}

This research is partially supported through projects of the Spanish Ministry of Economy and Competitiveness with Ref: TIN2010-21272C02-01 (funded by the European Regional Development Fund), and SA405A12-2 from Junta de Castilla y León. This work was also supported in the framework of the IT4 Innovations Centre of Excellence project, Reg. no. CZ.1.05/1.1.00/02.0070 by operational programme 'Research and Development for Innovations' funded by the Structural Funds of the European Union and state budget of the Czech Republic, EU.

Guest Editors

Emilio Corchado*

Departamento de Informática y Automática, Universidad de Salamanca, Plaza de la Merced S/N 37008, Salamanca, Spain IT4Innovations, Center for Excelence, VSB-Technical University of Ostrava, 17. Listopadu 15, 70833 Ostrava, Poruba, Czech Republic

E-mail address: escorchado@usal.es

Ajith Abraham

IT4Innovations, Center for Excelence, VSB-Technical University of Ostrava, 17. Listopadu 15, 70833 Ostrava, Poruba, Czech Republic Machine Intelligence Research Labs (MIR Labs), Scientific Network for Innovation and Research Excellence, P.O. Box 2259,

Auburn, WA 98071, USA

E-mail address: ajith.abraham@ieee.org

Received 28 October 2013

\footnotetext{
* Corresponding author at: Departamento de Informática y Automática, Universidad de Salamanca, Plaza de la Merced S/N 37008, Salamanca, Spain. Tel.: +34630736755.
} 